\title{
Histological changes in the human prostate after radiotherapy and salvage high intensity focused ultrasound
}

\author{
Venu Chalasani, MD; Carlos H. Martinez, MD;* Andrew K. Williams, MD;* Kevin Kwan, MD; Joseph L. Chin, \\ MD, FRCSC ${ }^{*}$
}

\begin{abstract}
The histological changes (both macroscopic and microscopic) in the prostate following the combination of external beam radiotherapy and salvage high intensity focused ultrasound (HIFU) have not been previously described. This article describes the case of a 65 -year-old male who presented with recurrent localized prostate cancer after undergoing external beam radiotherapy for low-risk prostate cancer. He was treated with salvage HIFU, and 4 weeks later presented with symptoms and signs consistent with a prostatorectal fistula. During a period of conservative management, his serum prostate-specific antigen levels started rising after having reached a nadir. A radical cystoprostatectomy and repair of fistula were performed after conservative management failed. Histological changes of dense fibrosis were noted in the region where the prostate should have been located. A literature review of the histological findings in the prostate after HIFU is discussed in this article, as well as the available evidence for the management of patients with local failure after the combination of external beam radiotherapy and salvage HIFU.
\end{abstract}

Can Urol Assoc J 2010;4(4):E100-102

\section{Case report}

A 65-year-old male received external beam radiotherapy for localized prostate cancer. His pre-radiotherapy prostate cancer characteristics were low risk (Gleason grade 6; clinical stage T1c, with a prostate-specific antigen [PSA] of $7 \mu \mathrm{g} / \mathrm{L}$ ). After radiotherapy, the PSA nadir was $0.9 \mu \mathrm{g} / \mathrm{L}$. Subsequently, he developed PSA failure, with the PSA rising to $5.05 \mu \mathrm{g} / \mathrm{L}$. A repeat transrectal ultrasound guided prostatic biopsy showed recurrent adenocarcinoma of the prostate in the left base. A metastatic workup was negative. The patient was treated with salvage high-intensity focused ultrasound (HIFU) as part of a phase II trial, using the Sonablate 500 Console (Focus Surgery, Inc., Indianapolis, IN). Rectal stenosis, needing dilatation, was noted. A suprapubic catheter was placed at the time of the procedure, and was removed at day 21.

About 1 month after the salvage HIFU treatment, the patient developed symptoms and signs consistent with a prostatorectal fistula, which was confirmed endoscopically. A urethral Foley catheter was placed to attempt healing by conservative measures in the first instance. The fistula did not heal with conservative measures alone. Unfortunately, the patient developed PSA recurrence during this time period. Metastatic workup remained negative. The patient was reluctant to have a defunctioning colostomy, and continued with Foley drainage.

After extensive discussions over the various options available for treatment, the likely consequences of previous radiotherapy and HIFU to the pelvic tissues, and the possibility of residual adenocarcinoma impairing healing, the patient underwent a cystoprostatectomy, formation of ileal conduit, debridement and repair of the rectal defect, pelvic lymph node dissection and formation of diverting sigmoid loop colostomy. Intra-operatively the prostate was not palpable; dense fibrotic tissue was noted between the bladder neck and the urethra, in the region where the prostate should have been located.

Macroscopic examination of the cystoprostatectomy specimen revealed a fistula tract in the posterior wall of the bladder. Careful dissection of the region, where the prostate should have been located, revealed fibrosed seminal vesicles and dense fibrous tissue. No prostate was identified. Microscopic sections of this area revealed atrophic viable seminal vesicles and dense fibrous connective tissue with no benign or malignant prostatic glands identified. The dense fibrous connective tissue showed reactive changes including fibroblast proliferation, neuronal proliferation and chronic inflammation (Fig. 1) (Fig. 2). These changes would be secondary to the prior treatment. There was no evidence of residual cancer. No evidence of malignancy was noted in the pelvic lymph nodes. The patient made an uneventful recovery; however, he has not undergone colostomy reversal as of yet due to anorectal manometry findings which revealed impairment of internal and external sphincter tone. This places him at higher risk of fecal incontinence. Serum PSA levels following cystoprostatectomy have dropped to $1.45 \mu \mathrm{g} / \mathrm{L}$. 


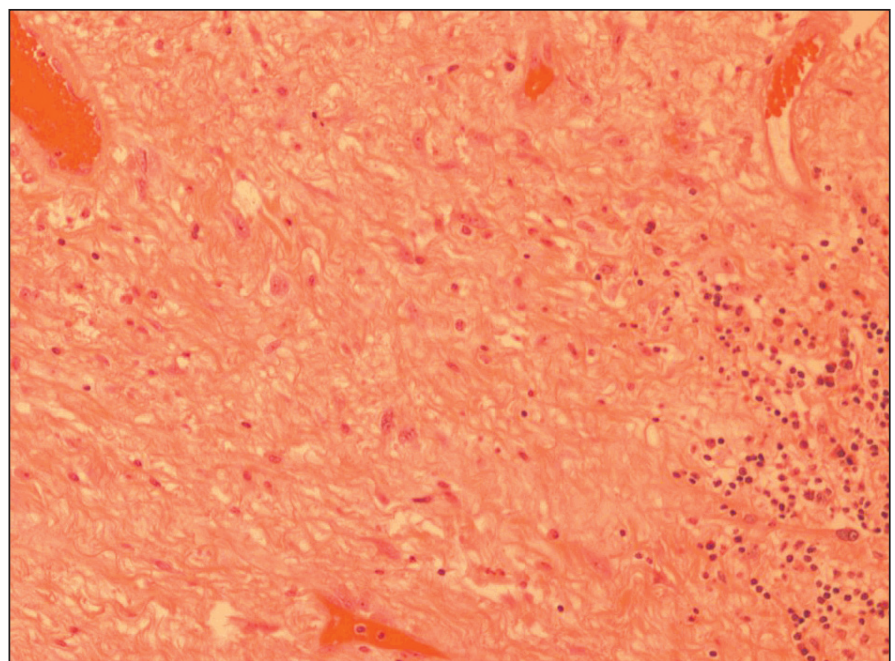

Fig. 1. High power magnification of the dense fibrous connective tissue with reactive changes and showing fibroblast proliferation, with reactive nuclear atypia, vascular proliferation and chronic inflammation.

\section{Discussion}

A recent study from the CaPSURE (Cancer of the Prostate Strategic Urologic Research Endeavor) database has revealed that PSA recurrence after radiotherapy occurred in $63 \%$ of men, ${ }^{1}$ with most patients (93.5\%) being treated with androgen deprivation therapy for salvage of PSA failure. The failure rate of radiotherapy has spurred interest in methods for salvage of PSA failure, such as salvage prostatectomy, ${ }^{2}$ salvage cryotherapy ${ }^{3}$ and salvage HIFU. ${ }^{4}$

High-intensity focused ultrasound is one of the minimally invasive treatment options available for localized prostate, with reports of its usage initially focusing on therapy for benign prostatic hyperplasia., ${ }^{5,6}$ Previous reports on the histopathological findings after HIFU have noted coagulative necrosis with sharply defined margins ${ }^{7,8}$ in cases where the patients underwent primary HIFU followed immediately by radical prostatectomy. Van Leenders and colleagues performed unilateral HIFU followed by radical prostatectomy, and noted mostly necrosis in the specimen, with electron microscopy revealing disintegration of cellular membranes and organelles. ${ }^{9}$ However they also noted prostatic epithelial glands in the HIFU-treated area with no evidence of necrosis, raising the possibility of incomplete treatment; these glands did not express cytokeratin, ${ }^{8}$ which is normally indicative of severe cellular disruption. ${ }^{9}$

Recently, there have been increasing reports of transrectal HIFU as a salvage treatment after radiotherapy failure, 4,10 however there are no reports of the intra-operative findings or histological changes in the prostate, which have been both irradiated and treated with HIFU. We noted, intraoperatively, that the prostate was not palpable, and this case illustrates the intra-operative difficultly that may arise

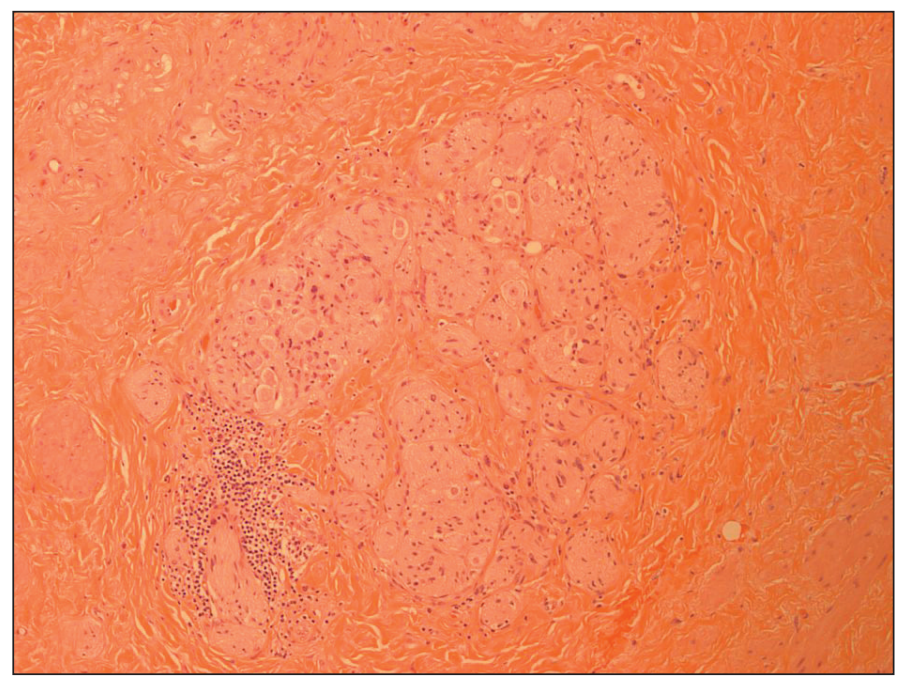

Fig. 2. Dense fibrous connective tissue with neuronal proliferation. Note that no prostatic glands are present.

for surgeons who are embarking on prostatectomy after the patient has failed the combination of radiotherapy and HIFU. Our case, in contrast to the aforementioned cases, showed dense fibrosis histologically, with no evidence of viable or necrotic prostatic glands, indicative of the likely severe biological insult to the prostate from the initial radiotherapy with subsequent HIFU. This case serves to illustrate the fine balance that exists between obtaining a histologically ablative prostatectomy and the complications that may ensue.

The management of patients who develop PSA failure subsequent to primary radiotherapy with salvage HIFU is uncharted. There are no case series reports in the literature of radical prostatectomy for this group of patients, leaving minimally invasive options or androgen deprivation therapy as the preferred options. One possibility is the usage of repeat HIFU, as described by Murat and colleagues, who reported treating 27 radiotherapy failure patients with a second session of salvage HIFU. ${ }^{10}$ Longer term follow-up is needed to clarify the outcomes for this group of patients who develop PSA recurrence following primary radiotherapy and salvage HIFU. Our pathological findings suggest that the PSA failure following salvage HIFU was likely metastatic in nature; unfortunately, there are currently no diagnostic tools which are sufficiently accurate to confirm this when serum PSA levels are low; the dilemma remains for physicians as to whether to offer definitive local treatment or whether to offer systemic treatment in the form of androgen deprivation therapy. Newer modalities of imaging, such as magnetic resonance imaging with superparamagnetic nanoparticles, ${ }^{11}$ may facilitate the detection of occult metastases in patients being considered for repeat salvage therapies, although it remains to be seen whether these techniques are widely adopted in clinical practice. 
Chalasani et al.

*Departments of Surgery and Oncology, Divisions of Urology and Surgical Oncology, Schulich School of Medicine \& Dentistry, London, ON; 'Department of Pathology, Schulich School of Medicine \& Dentistry, London, ON

Competing interests: None declared.

This paper has been peer-reviewed.

\section{References}

1. Agarwal PK, Sadetsky N, Konety BR, et al; and the Cancer of the Prostate Strategic Urological Research Endeavor (CaPSURE). Treatment failure after primary and salvage therapy for prostate cancer: likelihood, patterns of care, and outcomes. Cancer 2008;112:307-14.

2. Kaouk JH, Hafron J, Goel R, et al. Robotic salvage retropubic prostatectomy after radiation/brachytherapy: initial results. BJU Int 2008;102:93-6.

3. $\mathrm{Ng} C K$, Moussa M, Downey DB, et al. Salvage cryoablation of the prostate: followup and analysis of predictive factors for outcome. J Urol 2007;178:1253-7.

4. Zacharakis E, Ahmed HU, Ishaq A, et al. The feasibility and safety of high-intensity focused ultrasound as salvage therapy for recurrent prostate cancer following external beam radiotherapy. BJU Int 2008:102:786-92
5. Madersbacher S, Kratzik C, Szabo N, et al. Tissue ablation in benign prostatic hyperplasia with high-intensity focused ultrasound. Eur Urol 1993;23(Suppl 1):39-43

6. Gelet A, Chapelon JY, Margonari J, et al. High-intensity focused ultrasound experimentation on human benign prostatic hypertrophy. Eur Urol 1993;23 (Suppl 1):44-7.

7. Susani M, Madersbacher S, Kratzik C, et al. Morphology of tissue destruction induced by focused ultrasound. Eur Urol 1993;23(Suppl 1):34-8.

8. Madersbacher S, Pedevilla M, Vingers $L$, et al. Effect of high-intensity focused ultrasound on human prostate cancer in vivo. Cancer Res 1995:55:3346-51.

9. Van Leenders GJ, Beerlage HP, Ruijter ET, et al. Histopathological changes associated with high intensity focused ultrasound (HIFU) treatment for localised adenocarcinoma of the prostate. J Clin Pathol 2000;53:391-4.

10. Murat FJ, Poissonnier L, Rabilloud M, et al. Mid-term results demonstrate salvage high-intensity focused ultrasound (HIFU) as an effective and acceptably morbid salvage treatment option for locally radiorecurrent prostate cancer. Eur Urol 2009;55: 640-7.

11. Harisinghani MG, Barentsz J, Hahn PF, et al. Noninvasive detection of clinically occult lymph-node metastases in prostate cancer. N Engl J Med 2003;348:2491-9.

Correspondence: Dr. Joseph Chin, Divisions of Urology and Surgical Oncology, University of Western Ontario, London, 0N N6A 5W9; joseph.chin@hhsc.on.ca 